\title{
Adenosine stress CMR with variable density spiral pulse sequences accurately detects CAD with minimal dark-rim artifacts
}

\author{
Michael Salerno ${ }^{1 *}$, Angela M Taylor², Yang Yang ${ }^{4}$, Sujith Kuruvilla², Craig H Meyer ${ }^{4}$, Christopher M Kramer ${ }^{3,2}$ \\ From 17th Annual SCMR Scientific Sessions \\ New Orleans, LA, USA. 16-19 January 2014
}

\section{Background}

Adenosine stress CMR perfusion imaging has numerous advantages over competing modalities for assessing CAD demonstrating high diagnostic and prognostic utility. However, adenosine stress CMR perfusion imaging is limited by motion-induced dark-rim artifacts (DRA) which may be mistaken for true perfusion abnormalities. We have previously demonstrated that a high-resolution variable-density spiral pulse sequence with a novel density compensation strategy reduces ringing artifacts in firstpass perfusion imaging. The purpose of this study was to assess the clinical performance of this new technique to detect obstructive coronary artery disease (CAD).

\section{Methods}

CMR perfusion imaging was performed during adenosine stress $(140 \mu \mathrm{g} / \mathrm{kg}-\mathrm{min})$ and at rest on a Siemens $1.5 \mathrm{~T}$ Avanto scanner in 41 subjects with chest pain scheduled for coronary angiography (CA). Perfusion images were acquired during injection of $0.1 \mathrm{mmol} / \mathrm{kg}$ Gd-DTPA at 3 short-axis locations using a saturation recovery (SR) interleaved variable-density spiral pulse sequence. Sequence parameters included: SR time $80 \mathrm{~ms}$, FOV $320-340 \mathrm{~mm} 2$, nominal resolution $2.0 \mathrm{~mm} 2,8$ spiral interleaves, FA 30, TR/TE $9 \mathrm{~ms} / 1 \mathrm{~ms}$. Cine and late gadolinium enhanced (LGE) images were also obtained. All subjects underwent CA following the CMR and significant stenosis was defined as $>50 \%$ by quantitative CA. Two blinded reviewers evaluated the spiral perfusion images for the presence of adenosine-induced perfusion abnormalities and assessed image quality using a 5 point scale $(1$ - poor to 5- excellent).

'Medicine, Radiology, and BME, University of Virginia, Charlottesville, Virginia, USA

Full list of author information is available at the end of the article

\section{Results}

The patients had a mean age of $62 \pm 9,68 \%$ were male, $51 \%$ had a smoking history $46 \%$ had diabetes, $78 \%$ had hypertension and $95 \%$ had hyperlipidemia. $39 \%$ of the patients had a known history of CAD and $22 \%$ of the patients had undergone prior PCI. The mean LVEF by CMR was $61 \pm 7 \%$, and 14 patients (34\%) had LGE in a CAD pattern. QCA demonstrated obstructive CAD 28 (68\%) of the subjects. Figure 1 shows stress and rest spiral perfusion images from a subject who had normal cardiac function and no LGE. A reversible perfusion defect is present in the anterior wall corresponding to a high grade lesion in the LAD at CA. For the detection of a $50 \%$ stenosis by QCA the average sensitivity, specificity, and accuracy of the two readers were $89.3 \%, 84.6 \%$, and $87.8 \%$ respectively with a positive predictive value and negative predictive value of $92.6 \%$ and $79 \%$ respectively. The average image quality score was $4.4 \pm 0.7$ with only one study with more than mild DRA. There was good inter-reader reliability with a kappa statistic of 0.67 .

\section{Conclusions}

This study is the first to clinically evaluate spiral pulse sequences for adenosine stress CMR. There are a number of advantages to spiral pulse sequences including high efficiency, high SNR efficiency, robustness to motion, and isotropic spatial resolution. We demonstrate that these sequences produce high quality images with minimal dark-rim artifacts and demonstrate high diagnostic accuracy for assessment of CAD.

\section{Funding}

AHA 10SDG2650038, NIH K23 HL112910-01, Siemens Medical Solutions. 


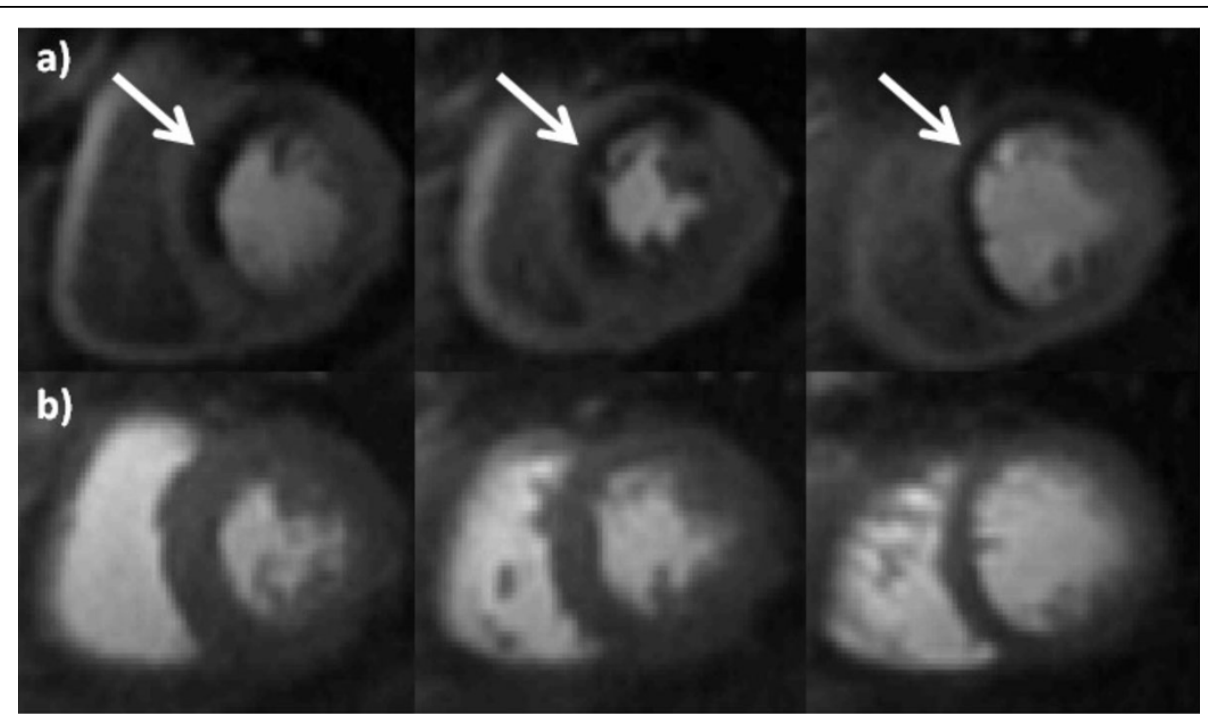

Figure 1 Stress (a) and Rest (b) adenosine-stress spiral perfusion images demonstrate a large anteroseptal perfusion defect corresponding with a high grade stenosis in the LAD at angiography. The images have high SNR and resolution with minimal DRA.

\section{Authors' details}

${ }^{1}$ Medicine, Radiology, and BME, University of Virginia, Charlottesville, Virginia, USA. ${ }^{2}$ Medicine, Cardiology, University of Virginia, Charlottesville, Virginia, USA. ${ }^{3}$ Radiology, University of Virginia, Charlottesville, Virginia, USA.

${ }^{4}$ Biomedical Engineering, University of Virginia, Charlottesville, Virginia, USA.

Published: 16 January 2014

\section{doi:10.1186/1532-429X-16-S1-058}

Cite this article as: Salerno et al:: Adenosine stress CMR with variable

density spiral pulse sequences accurately detects CAD with minimal

dark-rim artifacts. Journal of Cardiovascular Magnetic Resonance 201416

(Suppl 1):058.

\section{Submit your next manuscript to BioMed Central} and take full advantage of:

- Convenient online submission

- Thorough peer review

- No space constraints or color figure charges

- Immediate publication on acceptance

- Inclusion in PubMed, CAS, Scopus and Google Scholar

- Research which is freely available for redistribution 